\title{
REPRESENTING ROAD RELATED LASERSCANNED DATA IN CURVED REGULAR GRID: A SUPPORT TO AUTONOMOUS VEHICLES
}

\author{
V. Potó ${ }^{1 *}$ A. Csepinszky², Á. Barsi ${ }^{1}$ \\ ${ }^{1}$ Dept. of Photogrammetry and Geoinformatics, Budapest University of Technology and Economics, 1111 Budapest, Hungary - \\ (poto.vivien, barsi.arpad)@epito.bme.hu \\ ${ }^{2}$ NNG, 1037 Budapest, Hungary - andras.csepinszky@nng.com
}

\author{
Commission II, WG II/3
}

KEY WORDS: curved regular grid, road surface, terrestrial laser scanning, mobile mapping, autonomous vehicle, transformation

\begin{abstract}
:
The terrestrial and mobile laser scanning has become nowadays a mature technology applied in several technical and non-technical applications. The transportation infrastructure can be surveyed by these technologies in an excellent way, then 3D maps, fly-through videos and road furniture inventories can be derived among many other applications. The very detailed measurement and the realistic feature enable even to be used in games or simulators. This advantage was to be analyzed in vehicular simulation environment; the primary goal of the paper was to demonstrate a potential workflow and use case for such laser scanning data. The selected simulation package was the OpenCRG, which is being a component of OpenDRIVE-OpenCRG-OpenSCENARIO system, where it has been developed for microscopic simulations, e.g. vibrations, tire models or vehicle suspension systems. Because of the realistic visualization of CRG models it is very popular in the design and development of autonomous vehicles. The paper presents two different paved pilot sites surveyed by these technologies, then the raw data preparation is described and the details of the CRG model building is shown. The results of the experiments bring an overview, how the captured field data can be represented and interpreted in road surface context. The diagrams illustrate the potential of the very high resolution $(1 \mathrm{~cm})$ model, which allows to identify each separate cobble stone or to study surface roughness.
\end{abstract}

\section{INTRODUCTION}

One of the most developing field in the twenty-first century is the vehicle industry. A long dream of the mankind is having a road transportation system, where vehicles can move in fully automatic way. The self-driving vehicles are therefore a very often mentioned, not only in scientific, but also in all-day papers. The vehicle automation has been categorized by the International Society of Automotive Engineers (with well-known abbreviation SAE) by its standard J3016_201609 Taxonomy and Definitions for Terms Related to Driving Automation Systems for On-Road Motor Vehicles. The SAE categories distinguish between two basic groups; both have three subgroups, numbered from zero to five. SAE levels 0 to 2 represents vehicles, where human drivers monitor the environment and only driver assistants and partial automation are available. SAE levels 3 to 5 express when an automated driving system monitors the environment and conditional automation, high automation and full automation sublevels are there. Nowadays most developed vehicles are at about Level 3. (International Society of Automotive Engineers, 2016)

There is a common agreement that fully automated driving requires different technical achievements, like advanced sensory systems, advanced actuator system, suitable power supply system, reliable control system (Matthaei and Maurer, 2015). Most experts understand that the control system replaces really the human driving action, where decision making tasks have to be solved, most probably by the use of artificial intelligence. The best decisions are effectively supported by the newly defined map database, where the traditional static map content is extended by dynamic features (road works, weather information, traffic, congestion and accidents, traffic signals, even vehicles) (SIP-adus Automated Driving for Universal Service, 2018). The new map database stores significantly more geometric and attribute details about the environment, so the high-definition (HD) map term has been introduced in comparison to the smalldefinition (SD) map delivered for human applications.

The mentioned system components require further the capability of vehicular communication (called connected driving), where the vehicles can send and receive information to and from other vehicles and the infrastructure (Campolo, Molinaro and Scopigno, 2015).

The automotive development lifecycle has a strong integrated simulation phase, which become more importance nowadays. By the use of simulations, the car manufacturers can have a tool which enables faster prototype testing in more accurate way, more complex analyses are available and dominant cost reduction is feasible. The simulation environments have a very crucial requirement: they have to be realistic as far as possible. To achieve the most available realistic modelling scenario, real world data capture is strongly involved in the environmental preparation. This means for surveying and mapping, that the highly valued field measurement techniques can contribute within work. The goal of the authors was therefore to demonstrate how an automotive testing environment can be efficiently supported by laser scanning data capture. The selected simulation package is the OpenDRIVE - OpenCRG OpenSCENARIO triple, which aim to be a platform for driving analysis scenes, first of all in support of self-driving vehicle development.

The paper has the following structure. Section 2 presents a global view about the simulation environment. Section 3 covers the presentation of the pilot sites and the applied data capturing techniques, while Section 4 describes the workflow of building OpenCRG models from laser scanned data. The results are shown in Section 5 and the paper is finished with some concluding remarks in Section 6.

* Corresponding author 


\section{CURVED ROAD GRID AND OPENCRG}

The OpenDRIVE project has begun in 2005 as a German company, the VIRES Simulationstechnologie started building visual databases for various driving simulators and its customers helped understand, that every company needs the same road information unless they use different formats. So VIRES and Daimler Driving Simulator started to standardize them. It was published in 2006, later other big companies joined to this initiative, e.g. 3d Mapping Solutions, BMW Group, Deutsches Zentrum für Luft- und Raumfahrt (DLR), HERE Technologies. Today OpenDRIVE has become an international standard. OpenDRIVE contains the logical description of the road network. It has an own file format, the OXDR with a hierarchical structure and is available also in XML format, which helps the data exchange between different data types. It contains also the road neighborhood; the main parts are the road segments, the junctions and the controllers. Roads are described by their center line. The geometry has three main elements: line, spiral and arc. Data is collected from road scans, navigation data, road network design software, etc. OpenDRIVE can be used in traffic and sensor simulations. (OpenDRIVE webpage, 2015)

The OpenCRG project has started in 2008. The aim of the project is providing open format and tools for detailed road surface description. OpenCRG is useful for tire simulations, vibration and driving simulations. The first user was Daimler AG. By the combination of OpenDRIVE and OpenCRG a realistic static test field can be established.

CRG means "curved regular grid". It refers to the road section description method. The global coordinate system with $x$ and $y$ coordinates are transformed into a regular grid, as it is shown in Fig. 1.

This grid has a $u$ axis, that means the road reference line, and $v$ coordinates are perpendicular to the $u$ axis. The grid cells contain height information about the road surface. OpenCRG provides Matlab and $\mathrm{C}$ tools for creating, handling, manipulating and evaluating the CRG files. (OpenCRG webpage, 2016)

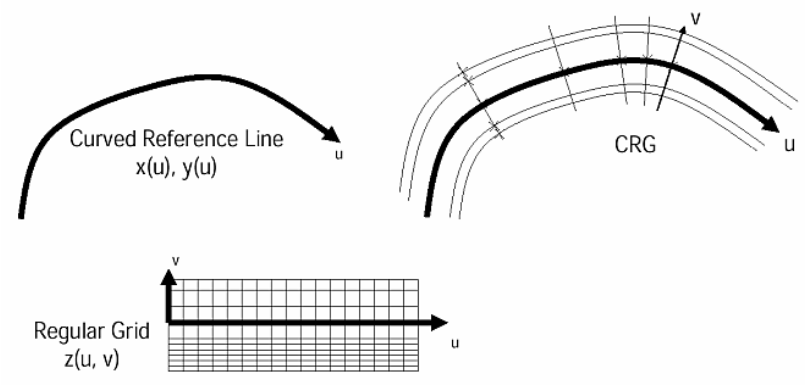

Figure 1. Creating curved regular grid

(OpenCRG webpage, 2016)

OpenSCENARIO is the newest member of the OpenX family. It has started in 2014, but it is still in the "under construction" state. It deals with the simulation's dynamics contents, like traffic, congestion etc. (OpenSCENARIO webpage, 2017)

\section{FIELD DATA CAPTURE}

There were two pilot sites selected in order to be able to present the variability of the urban scenarios and of course the terrestrial and mobile laser scanning can be parallel demonstrated. The terrestrial laser scanning (TLS) pilot site is in the campus of the Budapest University of Technology and Economics. It has a straight line segment having a length of about $100 \mathrm{~m}$. The second pilot site is measured by mobile mapping technology in the downtown of Budapest, it has two rectangular street segments forming a big $L$ shape. This site can demonstrate if the road axes turns by a fillet element (an arc). The total length of the pilot road is about $250 \mathrm{~m}$.

\subsection{Terrestrial laser scanning}

The terrestrial laser scanning (TLS) was executed in April 2017 by a Faro Focus 3D 120S scanner with the capability of horizontal viewing angle of $360^{\circ}$ and measuring distance of $120 \mathrm{~m}$. The geometric resolution was set for $6 \mathrm{~mm}$ in $10 \mathrm{~m}$. Because the primary goal was to survey the road surface in 3 dimension in a certain part of the campus, the image collection option was switched off and solely intensity values were stored. In order to remain in the acceptable measurement tolerance, several stations were set. 33 stations were needed for the laser scanning survey of the whole pilot site.

To be able to georeference the point cloud GNSS measurements were taken in 11 points by a Leica Viva CS10 controller and GS08 antenna unit. The measurement was done in RTK mode, where the correction was received from the Hungarian GPS permanent station network. The mean positioning error was $0.018 \mathrm{~m}$.

After the field work, the station point clouds were unified by the help of easily and uniquely identifiable terrain points. The merged point cloud contains 129 million points and requires 1.9 GB storage. The archiving was done in LAS format, but converted for the later use into binary PLY.

The continuation of the data clearing was the removal of disturbing objects: moving pedestrians, vegetation etc. Then the point cloud was cut keeping only a moderate height band above the road surface (Fig.2).

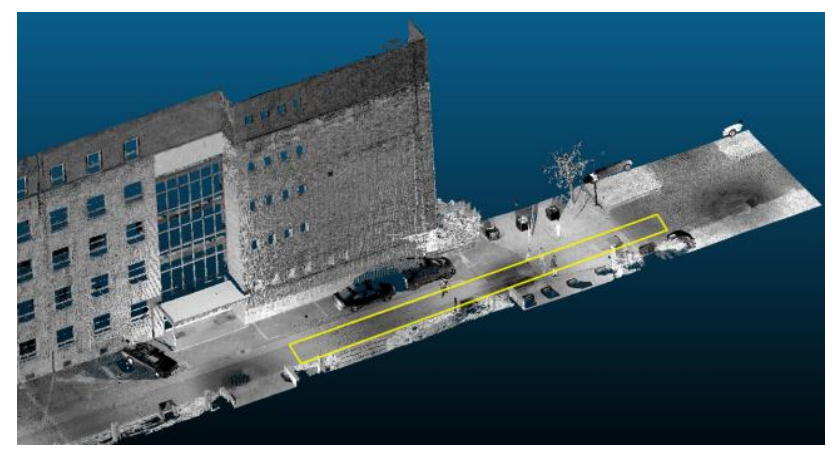

Figure 2. The analyzed road surface visualized with the TLS captured point cloud

\subsection{Mobile mapping}

The mobile laser scanning was executed by the Budapest Transportation Center (BKK) running a Riegl VMX-450 mobile mapping system. The system was equipped by two profile scanners, six cameras, GPS/INS receiver and a control unit. The point density varies between 0.5 and $3 \mathrm{~cm}$. The data capture was in May 2013. The GPS/INS integration (the trajectory computation) and then the point cloud was previously processed by the BKK; we have got the georeferenced version. Thanks to the continuously working cameras, the obtained point cloud was colored, although this information is still unneeded. The data was stored in LAS format and converted into binary PLY. The size of the file is $1.14 \mathrm{~GB}$ containing 29 million points.

As data preparation, the near road surface points were kept and others were removed from the file (Fig. 3). 


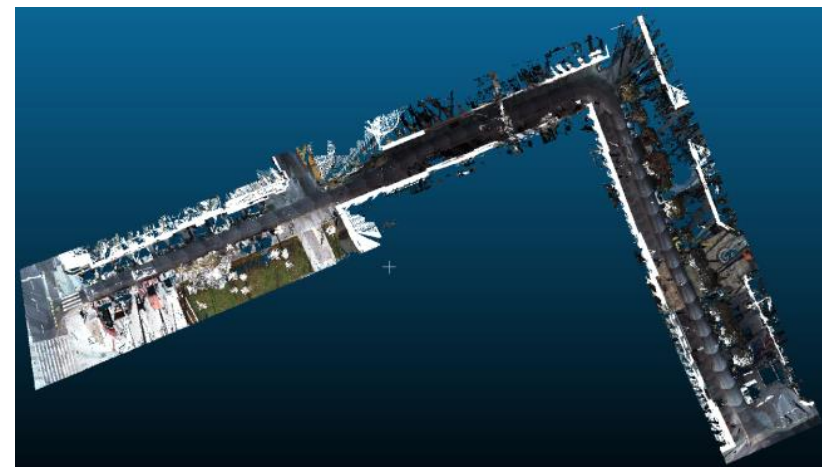

Figure 3. The MLS pilot site in the Budapest downtown (Right is the Váci street, left the Piarista street. The leftmost part is a square)

\section{BUILDING THE CRG-MODEL}

As it was shown in Section 2, OpenCRG uses the road geometry description of OpenDRIVE. This determines, that before any surface related action the core geometry has to be defined.

The TLS preparation means that the starting and ending reference point $x, y, z$ coordinates must set, the overall heading angle can then be derived. Further settings are the longitudinal and cross directional cell size, and the left and right road width must be given. In our case these two features were the same, meaning symmetric analyzed road surface. The pilot site was a first CRG realization, therefore no further slope and banking data were specified.

The MLS model preparation was started in a CAD system, where the road axes and the center point of the circular fillet was drawn. These points' coordinates (all $x, y$ and $z$ values) were stored in a matrix. The necessary settings were similarly the longitudinal and cross directional resolutions, the symmetric left and right road widths, and the lack of additional slope and banking information. By the definition of the longitudinal cell size, the heading angle vector can be computed.

The so defined model parameters are stored in the CRG model file. It was followed by the most computation intensive and time consuming phase, where each CRG cell has to be filled with elevation data. For a cell height value first its local $u$ and $v$ coordinates are to be transformed into the global (reference) coordinate system by a function being available in the CRG procedure library. The computed $x$ and $y$ coordinates can be taken as the center of a circle, where its radius limits the neighborhood for computing the mean height values from the laser scanned point cloud (Fig. 4) The average height is then stored in the CRG cell. The model building finishes with the storage of the whole model.

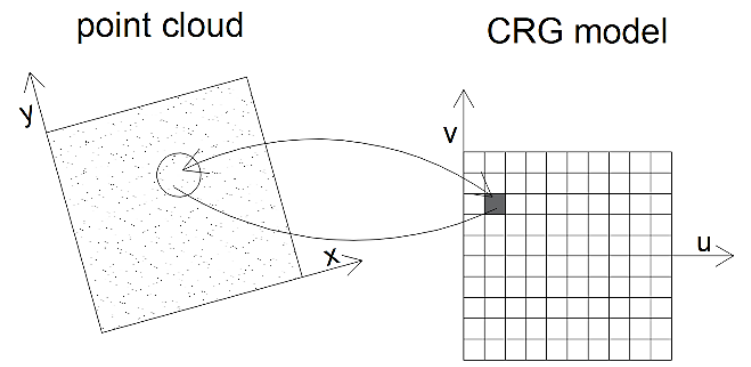

Figure 4. The projection of the CRG cell into the point cloud's global coordinate system with the neighborhood represented by the circle and the transfer of the mean height information back into the CRG cell
The coordinate transformation function handles any arbitrary global metric system; it can even be a local (projection-less) reference system.

The model building was performed in Mathworks Matlab R2017b.

\section{RESULTS}

The CRG model can be visualized by the use of the Matlab library. The results are twofold: there is an information page about the settings containing all parameters, comments and statistics about the features. The second result group is a series of diagrams:

- $\quad$ reference line maps

- reference line XY overview diagram with/without normalized negative curvature

$\circ$ reference line heading diagram

- reference line curvature diagram

- $\quad$ CRG elevation grid cuts and limits

- grid limits diagram

- CRG elevation grid cross sections

- CRG elevation grid longitudinal sections

- $\quad$ CRG road XYZ and UVZ map.

The MLS pilot site has a very common asphalt pavement, while the TLS pilot site was paved by concrete squared stone. Because the TLS site's surface is expected as more rough, the very high geometric resolution models, like $1 \mathrm{~cm}$ resolution models are more informative e.g. in grid detail diagrams. Fig. 5 shows longitudinal section diagram for a cut-off from the TLS pilot site.

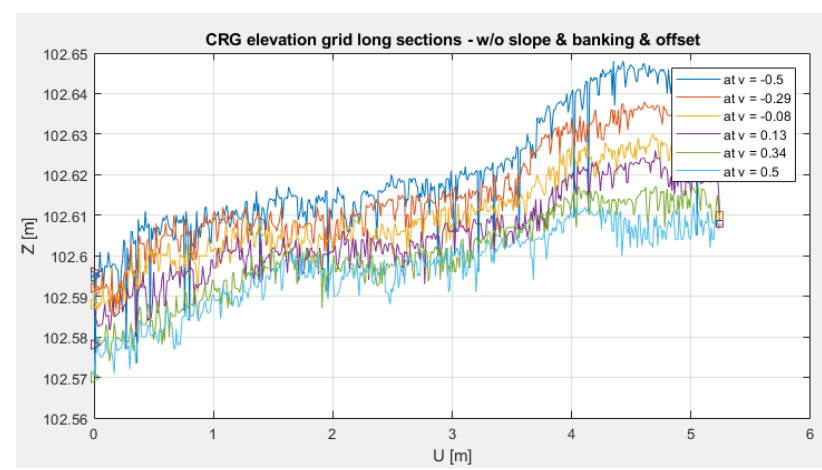

Figure 5. Longitudinal sections for a TLS data cut-off

As a trend it can be seen that the road section has a slight height increase, while the fine waves are due to the separate stones and the gaps (fugue) in between.

The grid visualization of the same cut-off can be seen in Fig. 6 . One can easily identify the height relations as well as the stones itself.

The whole TLS model with $1 \mathrm{~cm} u$ and $v$ resolution results a CRG grid having a grid size of $9410 \times 240$. It considers that the total length of the analyzed road was $94.1 \mathrm{~m}$ and the observed width was $2.4 \mathrm{~m}$. The pilot site is flat, the minimal and maximal height values were $102.224 \mathrm{~m}$ and $102.668 \mathrm{~m}$ respectively.

The MLS pilot site was longer, the total length was $259.55 \mathrm{~m}$. Because the asphalt pavement was in good condition, the very high resolution model has no meaning, so a $5 \mathrm{~cm}$ resolution model was created. Considering $1.5 \mathrm{~m}$ study width symmetrically on the left and right side of the road axis, the obtained grid size was $5192 \times 31$. The minimum height was $102.988 \mathrm{~m}$ and the maximum height had a value of $105.058 \mathrm{~m}$. 


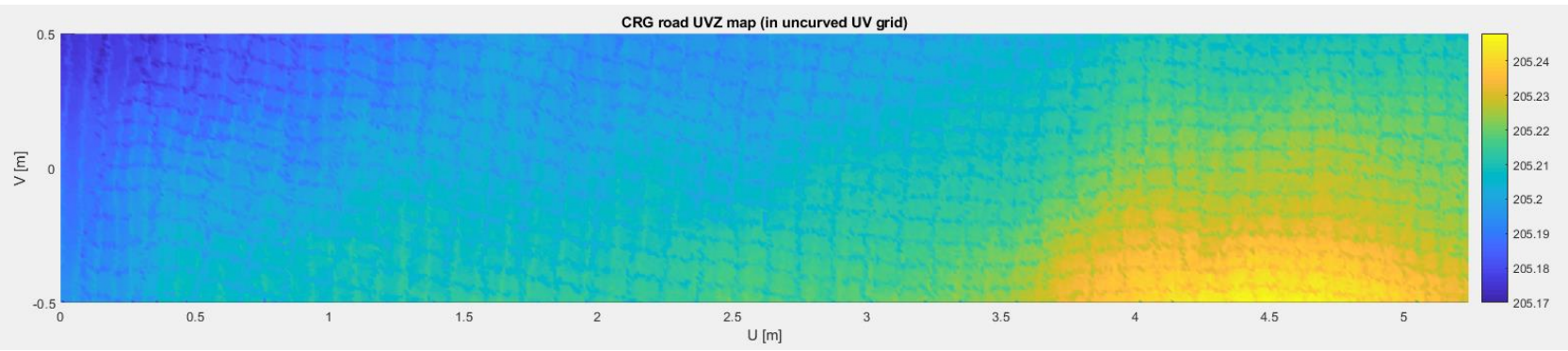

Figure 6. The grid map of the TLS pilot site

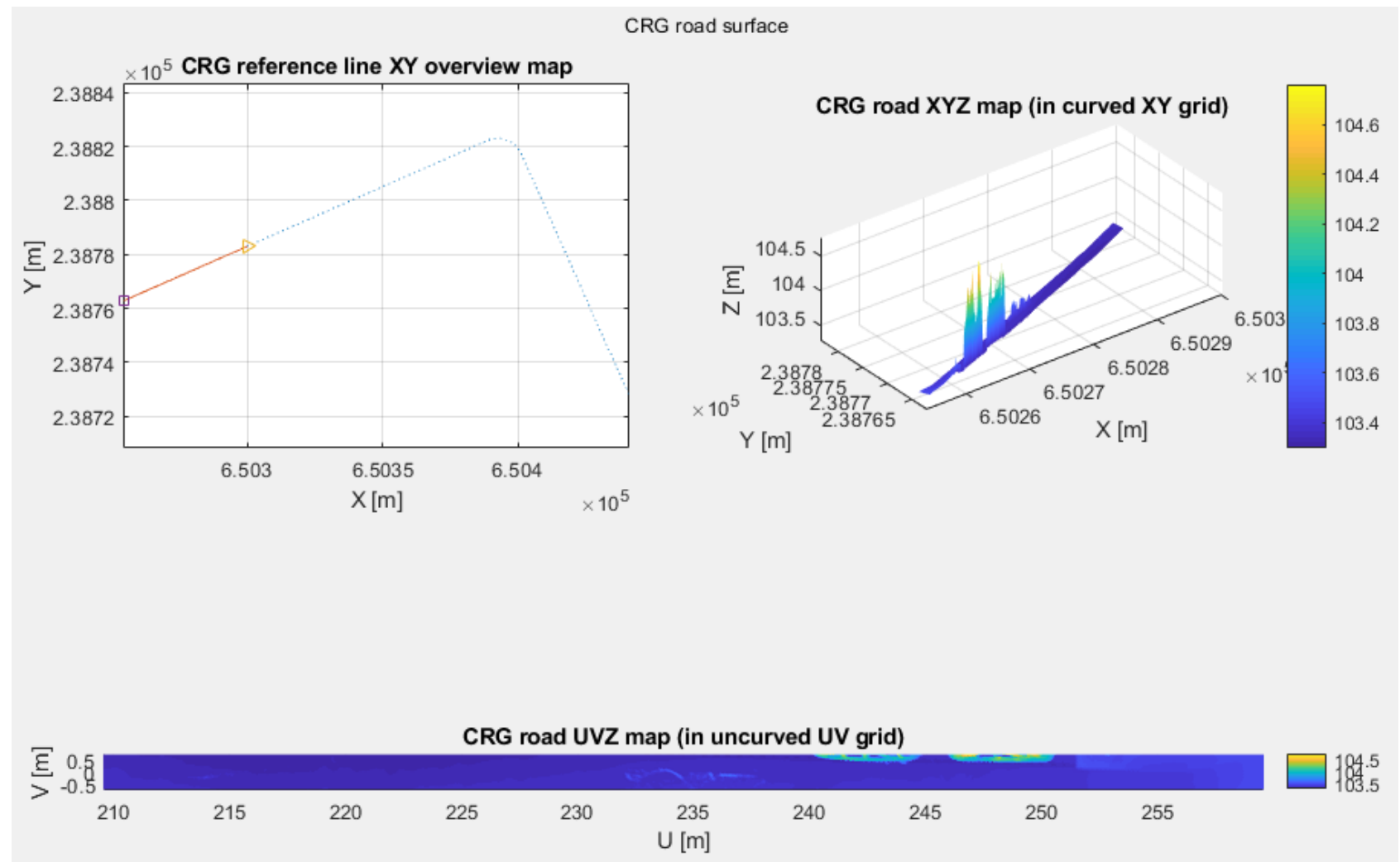

Figure 7. Example for the MLS pilot site visualization

Because the MLS pilot site has a longer road length, the finer details can only be visualized in parts. The available Matlab visualization tool of the CRG library ensures this functionality. Fig. 7 illustrates the last part of the pilot site. The first subplot (upper left) shows its position in the whole site, the 3dimensional plot in the upper right side presents the height values in very intuitive way, and the UVZ map (lower figure) indicates the grid.

It is clearly visible, that the MLS data set was not only cut from the road surface; instead, some small parts of the parking vehicles have also remained in the point cloud (two vehicles are easily to be recognized between 240 and $250 \mathrm{~m}$ ). The grid representation draws the attention on some road anomalies, which can be detected near $235 \mathrm{~m}$.

The CRG visualization tools bring information also about the road geometry; in our MLS pilot site the road curvature is worth to study. The intentionally selected pilot road has a near perpendicular segment, where a small radius curve ensures the continuity. The derived curvature figure clearly shows the increased values near the $u$ of $100 \mathrm{~m}$ (Fig. 8).

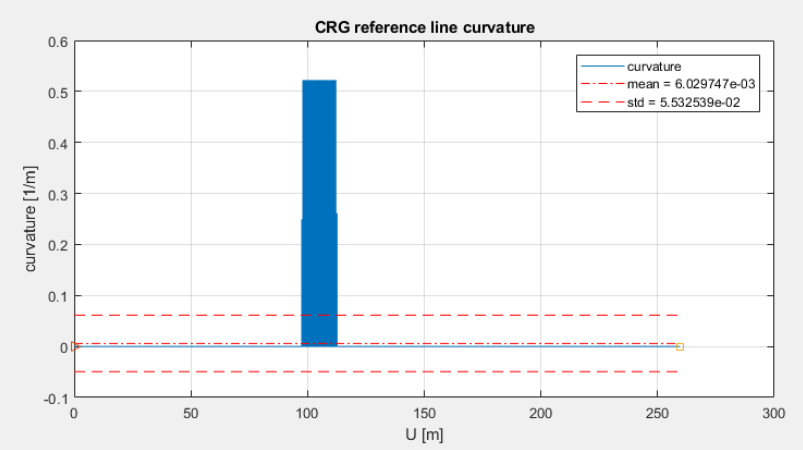

Figure 8. Curvature diagram for the MLS pilot site

The computational time for the $5 \mathrm{~cm}$ MLS model was $\sim 3$ hours on a laptop with Intel Core i $7700 \mathrm{HQ}$ processor and 16 GB RAM. This performance was achieved by the proof-of-concept run, but it can be significantly improved. 


\section{CONCLUSION}

The simulator software packages used in the vehicular development, like the OpenX family can describe the reality with high accuracy and very detailed. In order to fully utilize the ability of the software, it is desirable to feed with reality data instead of synthetic ones. The terrestrial and mobile laser scanning are excellent technologies for this purpose, which were proven in the current paper.

There were two differently paved pilot sites chosen and then surveyed by laser scanning. The $x, y, z$ coordinates of the point clouds were applied solely, the intensities or color information were ignored. After the point clouds being precisely prepared, a Matlab script was written to set the basic parameters of the CRG model and establish the coordinate transformation between the curved and the reality coordinate systems. All CRG cells were supplied by the adequate height information derived from the point clouds.

The methodology and the results have shown, that the widely used scanning methods suit for data capturing purposes of the simulator model creation. Several improvements are still to be achieved in the near future. These are the followings:

(1) The preparation of the laser scanned point clouds require strong human interaction: filtering and removal of noise and disturbing object has been done by human operators. By the implementation of high-precision and high-performance point cloud filtering techniques, this step would need less involvement of human operators.

(2) The geometry description of the road reference line is defined manually in the preparation phase. By the development of a software tool this step can be automatized and increased the productivity.

(3) The gridding technique which was written in Matlab has a lower performance (the script runs long even for cut-off data sets), which can be accelerated by the implementation based on the CRG C-API functionality.

The executed experiment demonstrates that the development of a more automatic point cloud processing workflow allure with the hope of producing highly detailed, realistic road infrastructure models, which has wide application fields, among them the autonomous vehicle development and testing can be supported effectively.

\section{ACKNOWLEDGEMENTS}

The project has been supported by the European Union, cofinanced by the European Social Fund. EFOP-3.6.3-VEKOP-162017-00001.

\section{REFERENCES}

Campolo, C., Molinaro, A. and Scopigno, R. (2015) 'From today's VANETs to tomorrow's planning and the bets for the day after', Vehicular Communications. Elsevier Inc., 2(3), pp. 158171. doi: 10.1016/j.vehcom.2015.06.002.

International Society of Automotive Engineers (2016) 'Surface vehicle recommended practice, J3016'.

Matthaei, R. and Maurer, M. (2015) 'Autonomous driving - A top-down-approach', At-Automatisierungstechnik, 63(3), pp. 155-167. doi: 10.1515/auto-2014-1136.
OpenCRG webpage (2016). Available at: http://www.opencrg.org/ (Accessed: 6 April 2018).

OpenDRIVE webpage (2015). Available at: http://www.opendrive.org/ (Accessed: 6 April 2018).

OpenSCENARIO webpage (2017). Available at: http://www.openscenario.org/ (Accessed: 6 April 2018).

SIP-adus Automated Driving for Universal Service (2018). Available at: http://en.sip-adus.go.jp/ (Accessed: 6 April 2018). 